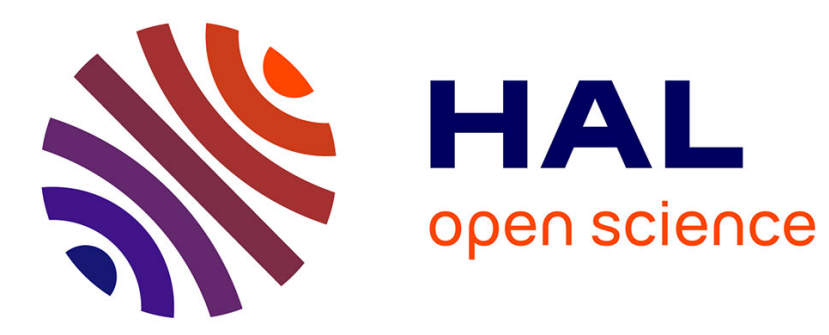

\title{
Analyse des interférences acoustiques
}

M. Servajean, B. de Raigniac

\section{To cite this version:}

M. Servajean, B. de Raigniac. Analyse des interférences acoustiques. Journal de Physique IV Proceedings, 1994, 04 (C5), pp.C5-1047-C5-1050. 10.1051/jp4:19945229 . jpa-00252915

\section{HAL Id: jpa-00252915 https://hal.science/jpa-00252915}

Submitted on 1 Jan 1994

HAL is a multi-disciplinary open access archive for the deposit and dissemination of scientific research documents, whether they are published or not. The documents may come from teaching and research institutions in France or abroad, or from public or private research centers.
L'archive ouverte pluridisciplinaire HAL, est destinée au dépôt et à la diffusion de documents scientifiques de niveau recherche, publiés ou non, émanant des établissements d'enseignement et de recherche français ou étrangers, des laboratoires publics ou privés. 


\title{
Analyse des interférences acoustiques
}

\author{
M. SERVAJEAN et B. DE RAIGNIAC
}

Société AERO, Allée Jean Giono, Chemin de Léry, 83500 La Seyne sur Mer, France

\begin{abstract}
Lofargrams of underwater acoustic field received on sono buoys often demonstrate interference phenomena due to beating between direct and surface reflected paths. Such deterministic differential phenomenon potentially conveys information on the source-receiver geometry. The direct problem of interference formation has been studied with the PARTAC model. In the general case, many ray-paths link source and receiver (eigen rays). An exhaustive analysis of time delays between the various ray-path families has led to comprehensive graphs. Observability criteria have been used to decipher those graphs to decide which interferences are visible. Solution of the inverse problem, i.e. determining the source-receiver geometry from the interference impulse response and grazing angles structure, has proved uneasy due to measurement difficulties and propagation limitations. Anyway if several rays from the same source can be identified, at least four including bottom returns, it is theoretically possible to determine the source depth and the distance to receiver.
\end{abstract}

\section{DESCRIPTION DU PHÉNOMÈNE D'INTERFÉRENCES ENTRE TRAJETS MULTIPLES}

Les lofargrammes sont des représentations temps-fréquence du signal acoustique reçu par des bouées ou des antennes passives qui peuvent présenter des phénomènes d'interférence. Il y a création d'un champ d'interférence chaque fois que le récepteur reçoit plusieurs rayons provenant d'une même source et ayant suivis des trajets différents (rayons directs, réfléchis surface ou fond, réfractés), qui n'ont donc pas les mêmes temps de propagation ni subi les mêmes déphasages. Le phénomène d'interférence résulte alors de l'addition cohérente des énergies des rayons à la réception.

Les interférences sont observées soit à courte distance (de l'ordre du kilomètre) à partir de bouées sur source profonde, soit à plus grande distance (une vingtaine de kilomètres) sur des antennes profondes pour les bruits rayonnés par des bâtiments de surface. Le phénomène est alors simple, puisqu'il se réduit à la combinaison des rayons direct et réfléchi surface. L'onde réfléchie à la surface paraît provenir d'une source image, symétrique par rapport à la surface, dont l'amplitude est à peu près égale à celle du signal direct et déphasée du décalage résultant de la différence de trajet et d'un déphasage de $\pi / 2$ à la réflexion. L'interférence est formée par l'addition cohérente des énergies des rayons direct et réfléchi surface, phénomène connu sous le nom de miroir de Lloyd.

Le spectre acoustique des signaux étudiés étant à large bande, les phénomènes d'interférences peuvent être observés sur l'ensemble du spectre. La décomposition en fréquence du signal reçu, coupe à un instant t du lofargramme, donne accès aux amplitudes résultant des interférences à chaque fréquence. Sur le 
lofargramme, l'addition algébrique des amplitudes des différents rayons forme des maxima (interférences constructives) et des minima (interférences destructives) locaux. Le contraste entre maxima et minima dépend des amplitudes relatives des deux rayons.

Le phénomène d'interférence est d'autant plus visible que les énergies des rayons sont voisines et que la distance source-récepteur varie au cours du temps : le lofar (figure 1) présente une famille de courbes homothétiques, lieux des maxima d'énergie et la courbe des retards, obtenue par autocorrélation.

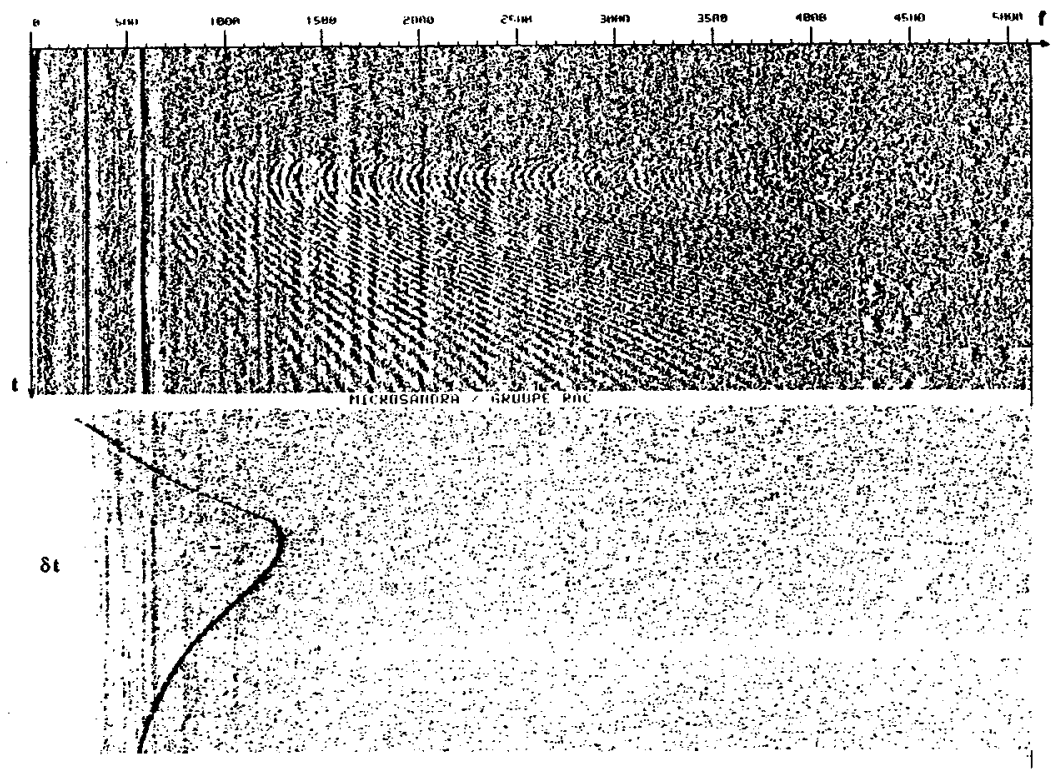

Figure 1 : Interférences entre 2 trajets observée sur un lofargramme et fonction d'autocorrélation

Le pas d'interférence $\Delta f$ est inversement proportionnel au retard $\Delta t$ entre les deux rayons produisant les interférences. Quand la distance source-récepteur évolue avec le temps, les retards sont modifiés, ainsi que le pas d'interférence. La distance source-récepteur diminuant, le réseau de lignes obliques quasi-parallèles évolue vers les basses fréquences, s'incurve et passe par un minimum au CPA (distance source-récepteur minimum). Au delà du CPA, il y a inversion de la pente et symétrie si la vitesse de la source est constante.

Les interférences sont caractéristiques de la géométrie source-récepteur à un instant donné et du milieu environnant. Ce phénomène différentiel, très sensible à certains paramètres d'évolution de la trajectoire du bruiteur, peut donc constituer un moyen de mesure, en particulier de limmersion et de la distance de la source acoustique observée.

\section{EXPLOITATION DU PHÉNOMĖNE D'INTERFÉRENCES}

Pour tenter d'exploiter ce phénomène déterministe et en tirer des informations sur le bruiteur, on a analysé le mécanisme générateur de ces interférences et examiné les possibilités d'en tirer des algorithmes permettant de déterminer la position (immersion, distance) de la source acoustique observée ${ }^{1}$.

Le modèle PARTAC, réalisé par Robert $\mathrm{LAVAL}^{2}$, a été adapté et utilisé pour l'analyse des phénomènes d'interférences. Le problème direct de la formation d'interférences par une source acoustique

\footnotetext{
${ }^{1}$ Contrat CERDSM N ${ }^{\circ}$ A9050063 "Analyse des interférence". Octobre 1992. Société AERO.

2 R. LAVAL, 1 nue Emile Duclaux, 75015 PARIS.
} 
large bande connue (immersion, vitesse) observée par une antenne fixe connue (immersion) a d'abord été étudié en fonction de la distance. PARTAC, très original par sa gestion rigoureuse des rayons, fournit les graphes des angles d'émission et de réception, d'anomalies de temps de propagation (ou temps réduits) et d'anomalies de pertes de propagation.

On a d'abord analysé les familles de paires de rayons se distinguant par une réflexion surface. Pour un couple d'immersions source et récepteur donné, l'organisation de ces retards en fonction de la distance, est stable, stucturée et cyclique (figure 2). On observe que les retards sont groupés en familles distinctes et tendent vers une valeur unique dépendant des immersions $Z_{1}$ et $Z_{2}$ de la source et du récepteur.

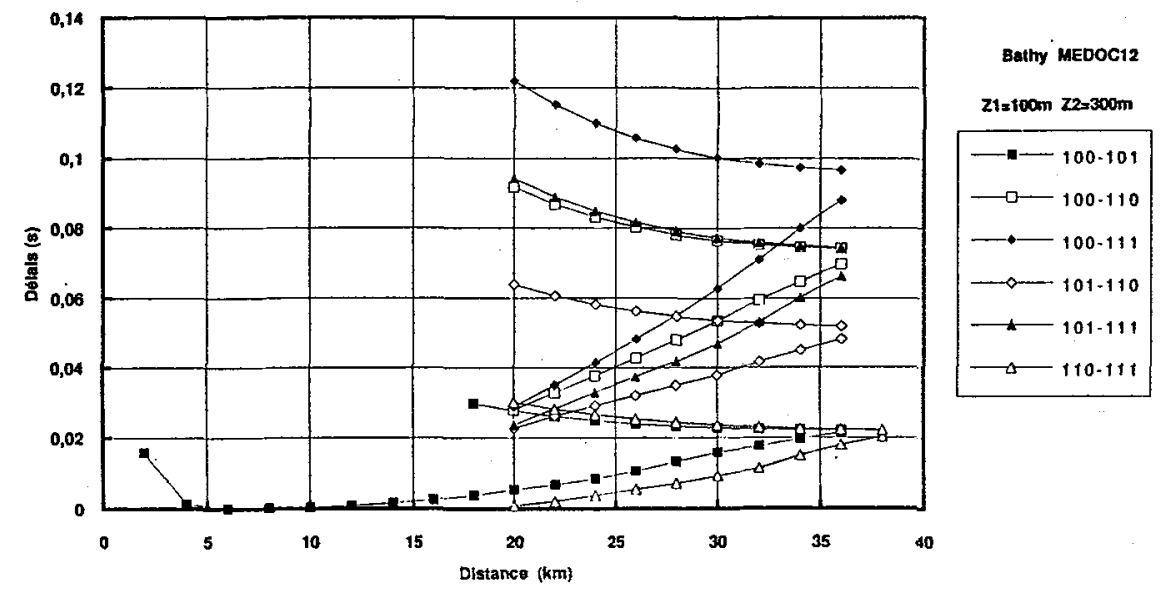

Figure 2 : Courbes de délais entre réfractés d'une part, et entre réfléchis fond d'autre part.

La gestion des rayons par associations deux par deux, devenant vite inextricable lorsqu'elle se veut exhaustive, une analyse systématique de toutes les combinaisons possibles de rayons, par la fonction d'autocorrélation, a été mise en œuvre. Elle a conduit à des figures de délais très complexes où tous les phénomènes sont représentés (figure 3).

Batny MEDOC12 "अronatio" 212200m 22=300m

Bathy MEDOC12"arrondio" $\quad z 4=200 \mathrm{mn} \quad 2=300 \mathrm{~m}$

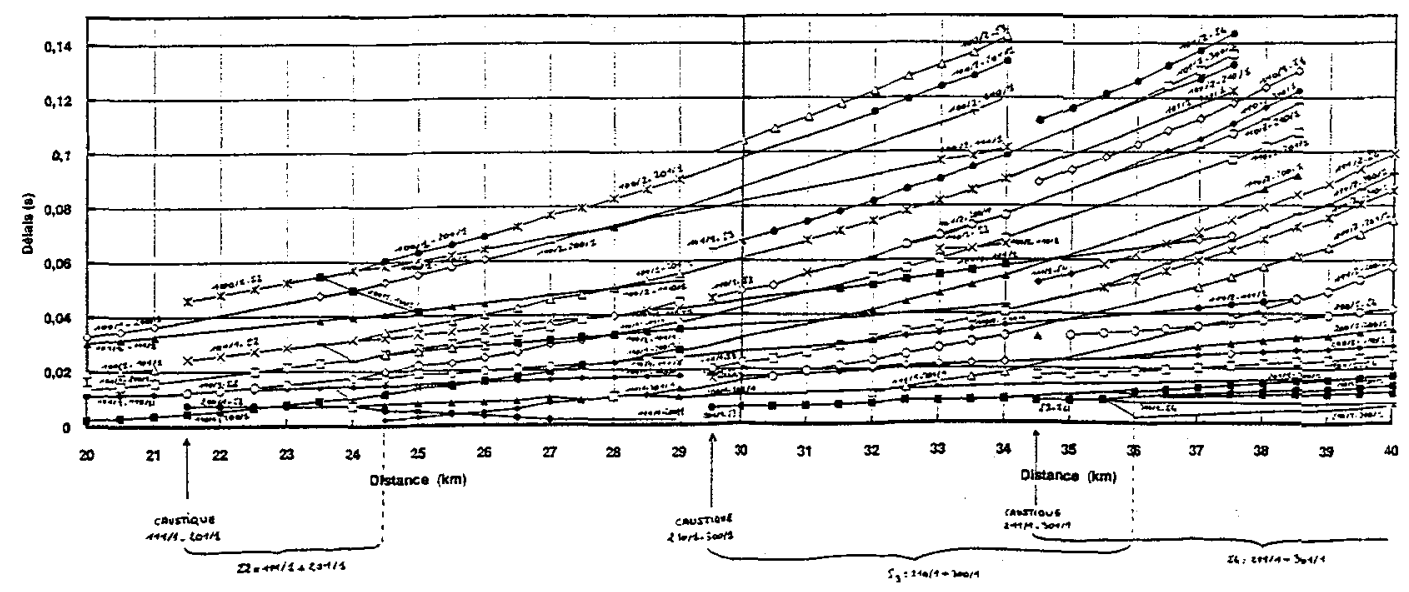

Figure 3 : Courbes de délais

Pour réduire la complexité de ces figures, on a étudié l'observabilité des interférences sur les lofar- 
grammes. Les énergies de chaque rayon ont été prises en compte et plusieurs critères, tels que la résolution temporelle des retards (bande passante utile), l'énergie de chaque interférence, le contraste minimal entre maxima/minima de chaque interférence, ont été définis pour sélectionner les interférences observables. Ils ont été insuffisants dans les situations compliquées car, vis à vis d'une interférence donnée, les autres interférences se comportent comme un bruit qui masque les minimas d'énergie. En présence de plusieurs interférences simultanées, il semble que seule une interférence de très forte énergie peut émerger, si elle existe.

\section{RÉSOLUTION DU PROBLÈME INVERSE}

L'analyse du problème inverse, i.e. la détermination de l'immersion et de la distance de la source acoustique à partir d'une réponse impulsionnelle théorique, suppose que l'observabilité des interférences a été résolue et permet de reconstruire la réponse impulsionnelle du milieu. La lisibilité et l'exploitation à des fins de localisation, de la fonction d'autocorrélation et de la réponse impulsionnelle associée, dépendent de la géométrie source-récepteur et du rapport signal sur bruit.

Avec une source profonde située à quelques dizaines de kilomètres, la réponse impulsionnelle comporte de nombreux rayons. Si cette réponse impulsionnelle permet d'en identifier plusieurs, au moins quatre avec si possible plusieurs réfléchis fond, et la mesure de leurs délais respectifs avec une bonne précision, il est possible d'évaluer l'immersion de la source acoustique, par une relation simple de proportionnalité (figure 4). Il est alors assez facile, connaissant les immersions de la source et du récepteur, de décuire la distance de la source par comparaison de la réponse impulsionnelle mesurée au graphe PARTAC des retards en fonction de la distance. L'évaluation de la distance peut comporter une ambiguïté d'une arche à une autre, qui devrait pouvoir être levée par l'examen de rayons suplémentaires.

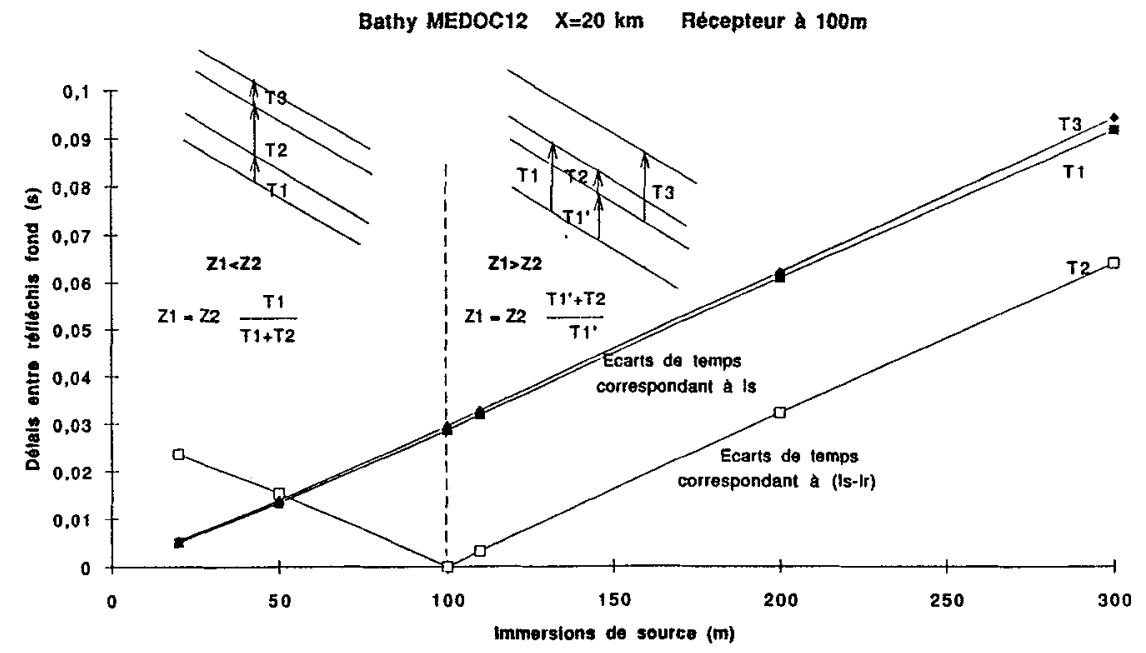

Figure 4: Variation des écarts de temps entre réfléchis fond en fonction de l'immersion de la source

La localisation de la source (immersion et distance) à partir de la mesure des retards et éventuellement des angles de site reste délicate en raison des difficultés liées aux mesures (retard, angle de site, doppler), des limitations dues aux pertes de propagation, au bruit et à la résolution temporelle des signaux et des ambiguïtés, en distance surtout, associées aux particularités de la propagation.

Le phénomène d'interférence peut donc conduire à la détermination de la position de la source acoustique. Pour cela, les interférences doivent être assez fortes (rapport S/B) et "riches" pour permettre d'isoler la réponse impulsionnelle du milieu de propagation, identifier plusieurs rayons et mesurer les retards associés. 